\title{
High altitude journeys, flights and hypoxia: any role for disease flares in IBD patients?
}

Vavricka, Stephan R ; Rogler, Gerhard ; Biedermann, Luc

\begin{abstract}
The importance of environmental factors in the pathogenesis including their disease-modifying potential are increasingly recognized in inflammatory bowel disease (IBD) patients, largely driven by the perception that the prevalence and incidence of IBD are on the rise within the last few years, especially in non-western countries. One of those factors is believed to be hypoxia. The role of hypoxia as a modifying or even causative factor in the genesis and maintenance of inflammation has been increasingly elucidated in recent years. Hypoxia is believed to be a main inducing factor of inflammation. This has been studied in different animal experiments as well as in humans exposed to hypoxia. In several studies - mainly in mice - animals exposed to short-term hypoxia accumulated inflammatory cells in multiple organs and showed elevated cytokines in the blood. Comparable studies were performed in humans, mainly in healthy mountaineers. Recently, we reported on the association between IBD flare-up episodes and antecedent journeys to high-altitude region and aircraft travels. According to these findings, we concluded that flights and stays at high altitudes of $>2,000 \mathrm{mg}$ are a risk factor for increased disease activity in IBD. To evaluate the potential influence of hypoxia on the course of IBD on a biomolecular level and to test the effects of hypoxia under standardized conditions, we initiated a prospective and controlled investigation in both healthy controls and IBD patients in stable remission. The study participants underwent a 3-hour exposure to hypoxic conditions simulating an altitude of 4,000 $\mathrm{m}$ above sea level in a hyperbaric pressure chamber and clinical parameters as well as blood and stool samples were collected at several time points. The first results of this study are expected in the near future.
\end{abstract}

DOI: https://doi.org/10.1159/000442932

Posted at the Zurich Open Repository and Archive, University of Zurich ZORA URL: https://doi.org/10.5167/uzh-134541

Journal Article

Published Version

Originally published at:

Vavricka, Stephan R; Rogler, Gerhard; Biedermann, Luc (2016). High altitude journeys, flights and hypoxia: any role for disease flares in IBD patients? Digestive Diseases, 34(1-2):78-83.

DOI: https://doi.org/10.1159/000442932 


\title{
High Altitude Journeys, Flights and Hypoxia: Any Role for Disease Flares in IBD Patients?
}

\author{
Stephan R. Vavricka ${ }^{a}$ Gerhard Rogler ${ }^{b}$ Luc Biedermann ${ }^{b}$ \\ a Division of Gastroenterology and Hepatology, Triemli Hospital, ' Division of Gastroenterology and Hepatology, \\ University Hospital, Zurich, Switzerland
}

\section{Key Words}

Inflammatory bowel disease - Crohn's disease - Ulcerative colitis - Oxygen supply · Hypoxia · Hypoxemia · High altitude Pressure chamber $\cdot$ Hypoxia-inducible factors . Inflammation · Autophagy · Inflammasome · Intestinal microbiota $\cdot$ Microbiome

\footnotetext{
Abstract

The importance of environmental factors in the pathogenesis including their disease-modifying potential are increasingly recognized in inflammatory bowel disease (IBD) patients, largely driven by the perception that the prevalence and incidence of IBD are on the rise within the last few years, especially in non-western countries. One of those factors is believed to be hypoxia. The role of hypoxia as a modifying or even causative factor in the genesis and maintenance of inflammation has been increasingly elucidated in recent years. Hypoxia is believed to be a main inducing factor of inflammation. This has been studied in different animal experiments as well as in humans exposed to hypoxia. In several
}

studies - mainly in mice - animals exposed to short-term hypoxia accumulated inflammatory cells in multiple organs and showed elevated cytokines in the blood. Comparable studies were performed in humans, mainly in healthy mountaineers. Recently, we reported on the association between IBD flare-up episodes and antecedent journeys to high-altitude region and aircraft travels. According to these findings, we concluded that flights and stays at high altitudes of $>2,000 \mathrm{mg}$ are a risk factor for increased disease activity in IBD. To evaluate the potential influence of hypoxia on the course of IBD on a biomolecular level and to test the effects of hypoxia under standardized conditions, we initiated a prospective and controlled investigation in both healthy controls and IBD patients in stable remission. The study participants underwent a 3-hour exposure to hypoxic conditions simulating an altitude of $4,000 \mathrm{~m}$ above sea level in a hyperbaric pressure chamber and clinical parameters as well as blood and stool samples were collected at several time points. The first results of this study are expected in the near future.

(c) 2016 S. Karger AG, Basel

\section{KARGER}

E-Mail karger@karger.com www.karger.com/ddi
(C) 2016 S. Karger AG, Basel

0257-2753/16/0342-0078\$39.50/0
Stephan R. Vavricka, MD

Division of Gastroenterology and Hepatology

Triemli Hospital, Zurich (Switzerland)

E-Mail stephan.vavricka@triemli.stzh.ch 


\section{Background}

The crucial role of hypoxia as an inductor of inflammation has been studied extensively in animal experiments as well as in humans exposed to hypoxia. In several studies - mainly in mice - animals exposed to shortterm hypoxia accumulated inflammatory cells in multiple organs and showed elevated cytokines in the blood [1-5]. Comparable studies were performed in humans, mainly in healthy mountaineers. One study, for example, investigated healthy volunteers who had spent 3 nights at an elevation of 3,400 $\mathrm{m}$ above sea level [6]. In those individuals, an increase of different cytokines such as IL-6, IL-6 receptor and CRP in blood was detected, suggesting an important role of hypoxia in inflammation. Other studies investigated the impact of hypoxia on intestinal transport proteins, sleep, nutrition, exercise capacity, lung function, lipid metabolism and gastric emptying in healthy mountaineers [7-9]. Furthermore, it is well known that exposure to high altitudes may lead to gastric and duodenal erosions and ulcer formation with consecutive gastrointestinal bleeding $[10,11]$.

Our own group performed a study in which healthy volunteer mountaineers first received an (unsedated) endoscopy at baseline in Zurich. With the sole exception of one subject with reflux esophagitis, all subjects revealed normal endoscopic findings. After that, the study participants went to an altitude of 4,500 above sea level, where 2 further endoscopies were performed. At day 2 (the time of the second endoscopy, performed in 18 subjects), abnormal endoscopic findings were observed in $28 \%$ of subjects including hemorrhagic gastritis/duodenitis in 1 subject and duodenal erosions/ulcers in 4 subjects. Strikingly at day 4 (time of third endoscopic examination), the number of endoscopic abnormalities was as high as $61 \%$, that is, 16 out of 23 patients showed signs of gastrointestinal inflammation such as erosions/ulcers in the duodenum or stomach, hemorrhagic gastritis/duodenitis and reflux esophagitis. These results clearly implicate that even in experienced mountaineers hypoxia has profound effects on the gastrointestinal epithelium already after a period of 2 and 4 days, respectively, with exposure to hypoxic conditions [12]. Hypoxia has also been studied as a disease modifying factor and pro-inflammatory stimulus in several disease states, including in IBD, rheumatoid arthritis and cancer [13-18]. Even with regard to solid organ transplantation, hypoxia and consecutive ischemia has been identified as an important driver of inflammation, which ultimately may lead to graft failure [19]. The inter-relationship between hypoxia and inflammation does not appear to be unilateral but rather reciprocal, in that not only hypoxia can promote inflammation but also represent a consequence of the latter, for instance by impairment of blood flow by tissue edema or formation of reactive oxygen species which in turn may lead to oxygen depletion [20]. In the gut, a considerably steep and unique oxygen gradient can be observed in high proximity between the bowel lumen presenting an almost anaerobe compartment toward the highly perfused and oxygenrich intestinal epithelium [21]. Thus, intestinal epithelial cells are continuously facing hypoxemia in the bowel lumen [20]. The metabolic integrity of the intestinal mucosa is regulated by different effectors, such as intestinal microbiota, blood perfusion to the intestine and tissue oxygenation. However, even in the physiological state, there is a considerable fluctuation in oxygen partial pressure, due to the significant shifts in tissue perfusion for instance in the postprandial state [20]. In general, epithelial barrier integrity and absorptive functions are regulated by oxygen and substantial metabolic shifts are to be observed at sites of mucosal inflammation, where nutritional supply and oxygen are depleted, with consecutive hypoxia, hypoglycemia and lactate accumulation resulting in a decreasing $\mathrm{pH}$ [21]. A variety of processes involved in the pathophysiology of inflammation are characterized by a high demand and consumption of oxygen, including cell migration to inflammatory foci [22], phagocytosis [23] and bacterial killing [24] (often mediated by the generation of reactive oxygen species).

\section{Key Messages}

As a sequel of decreasing oxygen concentration, the expression of hypoxia-inducible factors (HIFs), present in virtually all human cells, has been well-established. The cells adapt to hypoxia with help of this factor. It exists as a heterodimer with 2 known subunits: HIF- $1 \alpha$ or HIF-2 $\alpha$ and HIF- $1 \beta$. HIF is activated in hypoxic conditions but is entirely inactive when oxygen is present [25-28]. In hypoxic conditions, HIF subunits translocate to the nucleus, where they bind as heterodimers to a hypoxia response promoter element, inducing transcription of numerous genes, including those of nuclear factor $-\kappa \mathrm{B}(\mathrm{NF}-\kappa \mathrm{B})$ and toll-like receptors (fig. 1). In normoxaemic conditions, HIF is targeted by proteosomal degradation. Several effector targets responding to hypoxia have previously been identified, among them targets associated to the generation of ATP, oxidative stress, iron absorption and immune responses [29-36]. 
Fig. 1. Regulation of HIF-1 according to the presence of oxygen. In the presence of oxygen HIF-1a protein is hydroxylated and susceptible to binding to other proteins, such as the E3 ubiquitin ligase VHL (von Hippel Lindau) complex, ultimately leading to proteasomal degradation. In hypoxia, enzymes enabling VHL to bind to HIF$1 \alpha$ are inhibited, precluding attachment of VHL to HIF-1 $\alpha$, which results in stabilization of the latter. Stabilized HIF-1 $\alpha$ the may translocate to the nucleus of the cell, where binding with HIF- $1 \beta$ occurs. This binding results in the formation of a heterodimer, which binds to the hypoxia-responsive elements (HREs). The latter contains the core sequence 5'-(A/G)CGTG-3' of the HIF-responsive genes. Among the genes regulated by HRE are those involved in angiogenesis (to increase oxygen supply), metabolism or apoptosis.

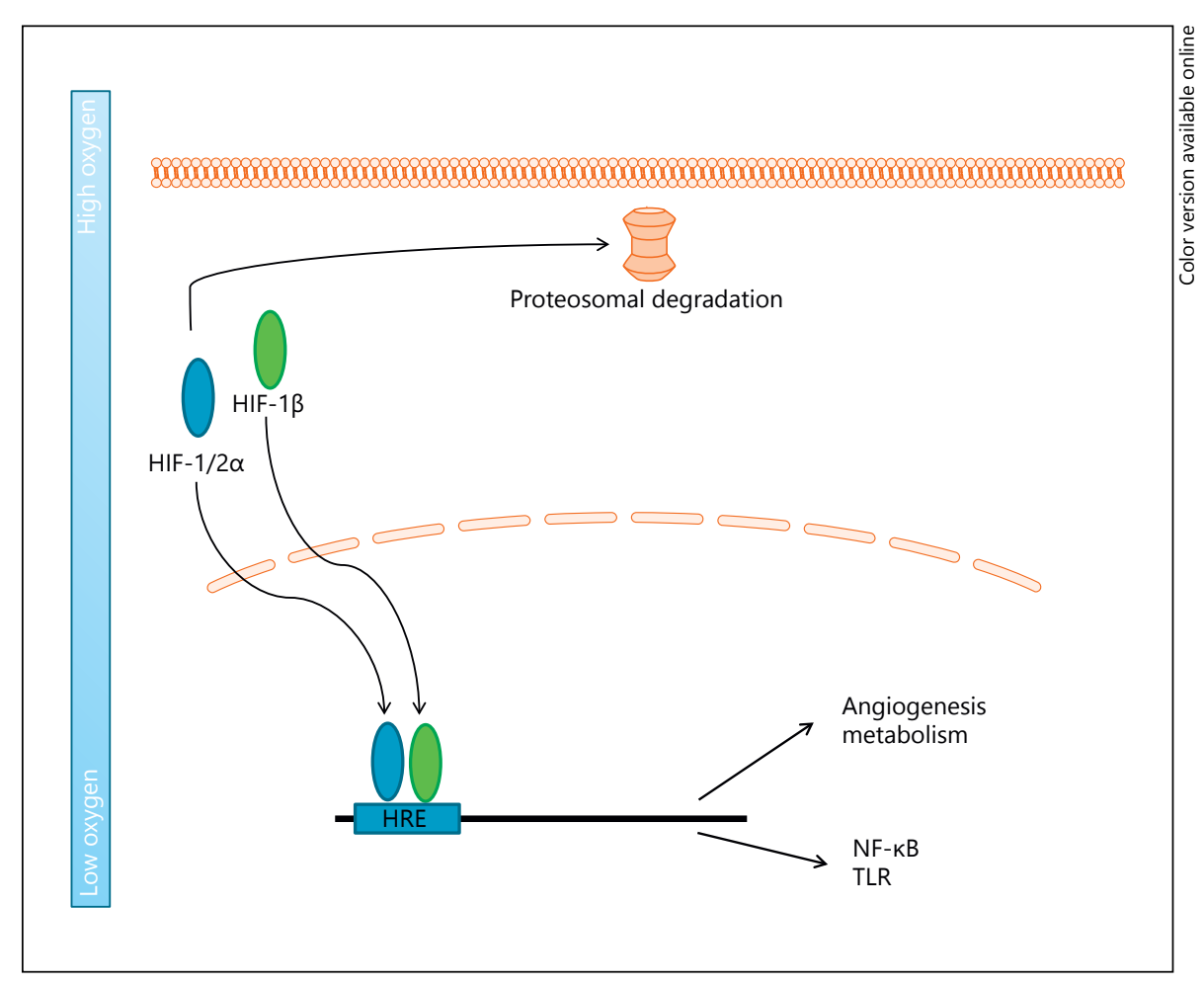

Inflammatory bowel disease (IBD) is a chronic, often debilitating intestinal disorder $[37,38]$. The etiology of IBD has not yet been fully elucidated; however, results of multiple studies point to a role of dysregulated innate and adaptive immune responses to intestinal bacteria as well as environmental factors, usually subsumized as exposome [39]. In IBDs such as Crohn's disease (CD) and ulcerative colitis (UC), HIF seems also to play a crucial role. Expression of HIF was found to be elevated in inflamed colonic mucosal biopsies of patients with $\mathrm{UC}$ and $\mathrm{CD}$ on the RNA level $[14,15]$ as well as on the protein level [16]. HIF appears to stimulate gene expression with regards to proteins involved in the maintenance of epithelial barrier integrity and function $[15,16]$. Thus, the transcriptional profile as a sequel of HIF expression ultimately exerts a protective role for the epithelium during inflammation. A mouse model with decreased expression of HIF-1 showed a more severe phenotype of colitis induced by trinitrobenzene sulfuric acid (TNBS), including an increased weight loss and symptoms as well as an increase in epithelial permeability and ultimately mortality, whereas overexpression of HIF revealed to be protective against the TNBS-induced damage [15]. Moreover, the $\mathrm{NF}-\kappa \mathrm{B}$, a key factor in the hyperactivation of effector immune cells in the pathogenesis of IBD, seems to be associ- ated to tissue oxygenation, as tissue ischemia and reperfusion was shown to promote its expression in mice [40]. With regard to general intestinal function, both intestinal absorption and permeability were shown to be modified by environmental oxygen concentration [41, 42]. Moreover, hypoxic conditions were shown to directly affect electrical currents when applied to the intestinal tissue from the serosa side [43], suggesting a direct effect of oxygen concentration on ion transport in the gut epithelium. Intriguingly, the induction of 'hypoxia-type' signaling by the inflammatory microenvironment in colitis has been observed, a phenomenon commonly referred to as 'inflammatory hypoxia' [20,44].

Stimulated by various reports from our IBD patients experiencing increasing disease activity after aircraft travel and high altitude stays, we initiated a retrospective study to investigate a potential epidemiological association between exposure to hypoxia and disease worsening in IBD [45]. In this retrospective study in 103 patients with IBD (50 with CD and 43 with UC), all participants were grouped into those previously having had a flare of disease (52 patients) versus those patients in sustained remission (51 patients). While in the former group $21 \mathrm{pa}-$ tients reported an aircraft travel or a high altitude sojourn (defined as above 2,000 m) within 4 weeks prior to the 
flare, only 8 patients stated such an exposure to high altitude in the latter group with sustained remission. This difference was significant in all IBD patients as well as CD patients, whereas a clear trend could be observed in those with UC. Interestingly, this association appeared to be even more pronounced in long-distant flights (i.e., those above $6 \mathrm{~h}$ ) as well as in high altitude stays of longer duration and those above 3,000 $\mathrm{m}$ [45]. Interestingly, at sea level, the oxygen fraction in the ambient air is roughly $21 \%$ with a pressure of $760 \mathrm{~mm} \mathrm{Hg}$. During airplane flights, the partial pressure of oxygen in the cabin is neither similar to the ambient pressure present on the altitude of the flight nor ground level, but reduced proportionally to the decrease in air pressure, which is technically maintained in the cabin and not permitted to fall lower than the ambient air pressure at 2,438 m $(8,000 \mathrm{ft}$; equaling $564 \mathrm{~mm} \mathrm{Hg}$ ), as required by international laws [46]. According to systematic investigations, the cabin air pressure during flight virtually always remains in the range between $1,524 \mathrm{~m}(5,000 \mathrm{ft}, 632 \mathrm{~mm} \mathrm{Hg})$ and the lowest permitted pressure (equaling 2,438 $\mathrm{m}$ ambient air) with a mean pressure value of $604 \mathrm{~mm} \mathrm{Hg}(1,894 \mathrm{~m})$ [47]. This increase in altitude (and decrease in oxygen partial pressure) corresponds to an inspirational oxygen fraction of $15.1 \%(2,438 \mathrm{~m})$ to $17.1 \%(1,524 \mathrm{~m})$ at sea level. Consecutively, this decrease in ambient oxygen partial pressure translates into a considerable decrease in the oxygen saturation in the peripheral blood ( 85 and $91 \%$, respectively) and mean arterial oxygen pressure (53 and $64 \mathrm{~mm}$ $\mathrm{Hg}$, respectively), nota bene in healthy volunteers [48]. In healthy subjects, this decrease in oxygen saturation is well tolerated in most instances, whereas hypoxia during aircraft travel may induce discomfort and medical complications in patients with pulmonary diseases [49].

While our study on increased activity in IBD patients after exposure to hypoxia [45] indeed revealed a significant association between inflammation and hypoxia, there are currently no clinical studies available in the literature. No studies have prospectively investigated a potential impact of hypoxia and hypoxemia on the clinical course of disease as well as biomolecular features. To evaluate the potential influence of hypoxia on the course of IBD on a biomolecular level and to test the effects of hypoxia under standardized conditions, we initiated a prospective and controlled investigation in both healthy controls and IBD patients in stable remission. Ten healthy volunteers, $10 \mathrm{CD}$ patients and $10 \mathrm{UC}$ patients underwent a 3-hour exposure to hypoxic conditions simulating an altitude of 4,000 $\mathrm{m}$ above sea level in a hyperbaric pressure chamber situated at the Swiss aeromedical centers in
Dubendorf, Switzerland. This study had been fully approved by the local ethics committee (EK 2013-0284). Stool samples analyzing calprotectin and microbiotal composition, biopsy samples from the rectosigmoid region and blood samples were repetitively collected and analyzed in conjunction with detailed records of clinical symptoms. At the moment, the study is closed and data are being evaluated. The first results are expected by the middle of 2016.

\section{Conclusion}

The importance of environmental factors in the pathogenesis including their disease modifying potential are increasingly recognized in IBD, largely driven by the perception that the prevalence and incidence of IBD are on the rise within the last few years, especially in non-western countries. Not only in these parts of the world, but also in Europe and North America, there have been substantial changes in environmental and life style factors, above all - but not exclusively - with regards to nutritional intake, often referred to as westernization, while the genetic pool has only modestly been subject to changes within that period. So far, the knowledge on the potential role of hypoxia in IBD is only scarce, despite both, numerous investigations in animal models and human, suggesting that hypoxia has a profound influence on the integrity and function of the gastrointestinal tract, epithelial barrier, immune system and microbial composition as well as the increasing body of evidence, that exposure to hypoxia (airline travel and high-altitude stopover) may be associated with various gastrointestinal symptoms and signs and furthermore has an impact on the course of disease in IBD.

Determining the mechanisms underlying these associations and the potential interplay of these factors are crucial to prevent and treat deleterious effects of hypoxia in IBD patients with future high-altitude sojourn. However, hypoxia and hypoxemia may indeed be occurring during exposure to ambient air with normal oxygen partial pressures. For instance, as a result of the altered physiology within the intestinal barrier in patients with IBD exposed to normobaric ambient air local hypoxemia is highly likely to be present, at least intermittently, such as for instance due to increased oxygen extraction as a result of altered metabolic properties in inflammation or impaired oxygen delivery due to alterations in vascular architecture as a sequel of tissue fibrosis, edema or antecedent surgical intervention. 
Thus, we consider our proposed research of importance for the general ('sea-level') IBD patient. Understanding molecular and microbiological consequences of intestinal hypoxia may ultimately derive further insights on the pathogenesis of IBD, far beyond exposure to lower partial oxygen pressure ambient air.

\section{Disclosure Statement}

The authors declare no disclosures.

\section{References}

1 Rosenberger P, Schwab JM, Mirakaj V, Masekowsky E, Mager A, Morote-Garcia JC, et al:Hypoxia-inducible factor-dependent induction of netrin-1 dampens inflammation caused by hypoxia. Nat Immunol 2009;10:195-202.

2 Eckle T, Faigle M, Grenz A, Laucher S, Thompson LF, Eltzschig HK: A2B adenosine receptor dampens hypoxia-induced vascular leak. Blood 2008;111:2024-2035.

3 Eltzschig HK, Ibla JC, Furuta GT, Leonard MO, Jacobson KA, Enjyoji K, et al: Coordinated adenine nucleotide phosphohydrolysis and nucleoside signaling in posthypoxic endothelium: role of ectonucleotidases and adenosine A2B receptors. J Exp Med 2003;198:783-796.

4 Thompson LF, Eltzschig HK, Ibla JC, Van De Wiele CJ, Resta R, Morote-Garcia JC, et al: Crucial role for ecto- $5^{\prime}$-nucleotidase (CD73) in vascular leakage during hypoxia. J Exp Med 2004;200:1395-1405.

5 Eltzschig HK, Abdulla P, Hoffman E, Hamilton KE, Daniels D, Schönfeld C, et al: HIF1 -dependent repression of equilibrative nucleoside transporter (ENT) in hypoxia. J Exp Med 2005;202:1493-1505.

6 Hartmann G, Tschöp M, Fischer R, Bidlingmaier C, Riepl R, Tschöp K, et al: High altitude increases circulating interleukin-6, interleukin-1 receptor antagonist and C-reactive protein. Cytokine 2000;12:246-252.

7 Wojtal KA, Cee A, Lang S, Götze O, Frühauf $\mathrm{H}$, Geier A, et al: Downregulation of duodenal SLC transporters and activation of proinflammatory signaling constitute the early response to high altitude in humans. Am J Physiol Gastrointest Liver Physiol 2014; 307:G673-G688.

8 Aeberli I, Erb A, Spliethoff K, Meier D, Götze $\mathrm{O}$, Frühauf H, et al: Disturbed eating at high altitude: influence of food preferences, acute mountain sickness and satiation hormones. Eur J Nutr 2013;52:625-635.

9 Siebenmann C, Bloch KE, Lundby C, Nussbamer-Ochsner Y, Schoeb M, Maggiorini M: Dexamethasone improves maximal exercise capacity of individuals susceptible to high altitude pulmonary edema at $4559 \mathrm{~m}$. High Alt Med Biol 2011;12:169-177.

10 Anand AC, Sashindran VK, Mohan L: Gastrointestinal problems at high altitude. Trop Gastroenterol 2006;27:147-153.

11 Wu TY, Ding SQ, Liu JL, Jia JH, Dai RC, Zhu DC, et al: High-altitude gastrointestinal bleeding: an observation in Qinghai-Tibetan railroad construction workers on Mountain Tanggula. World J Gastroenterol 2007;13: 774-780.

12 Fruehauf H, Erb A, Maggiorini M, Lutz TA, Schwizer W, Fried M, et al: T1080 unsedated transnasal esophago-gastroduodenoscopy at $4559 \mathrm{~m}(14957 \mathrm{ft})$ - endoscopic findings in healthy mountaineers after rapid ascent to high altitude. Gastroenterology 2010;138: S483-S484.

13 Thornton RD, Lane P, Borghaei RC, Pease EA, Caro J, Mochan E: Interleukin 1 induces hypoxia-inducible factor 1 in human gingival and synovial fibroblasts. Biochem J 2000; 350(pt 1):307-312.

14 Vermeulen N, Vermeire S, Arijs I, Michiels G, Ballet V, Derua R, et al: Seroreactivity against glycolytic enzymes in inflammatory bowel disease. Inflamm Bowel Dis 2011;17: 557-564.

15 Karhausen J, Furuta GT, Tomaszewski JE, Johnson RS, Colgan SP, Haase VH: Epithelial hypoxia-inducible factor-1 is protective in murine experimental colitis. J Clin Invest 2004;114:1098-1106.

16 Giatromanolaki A, Sivridis E, Maltezos E, Papazoglou D, Simopoulos C, Gatter KC, et al: Hypoxia inducible factor 1alpha and 2alpha overexpression in inflammatory bowel disease. J Clin Pathol 2003;56:209-213.

17 Shay JE, Imtiyaz HZ, Sivanand S, Durham AC, Skuli N, Hsu S, et al: Inhibition of hypoxia-inducible factors limits tumor progression in a mouse model of colorectal cancer. Carcinogenesis 2014;35:1067-1077.

18 Tsuzuki Y, Fukumura D, Oosthuyse B, Koike C, Carmeliet P, Jain RK: Vascular endothelial growth factor (VEGF) modulation by targeting hypoxia-inducible factor-1alpha - hypoxia response element - VEGF cascade differentially regulates vascular response and growth rate in tumors. Cancer Res 2000;60:62486252 .

19 Krüger B, Krick S, Dhillon N, Lerner SM, Ames S, Bromberg JS, et al: Donor toll-like receptor 4 contributes to ischemia and reperfusion injury following human kidney transplantation. Proc Natl Acad Sci U S A 2009; 106:3390-3395.

20 Colgan SP, Taylor CT: Hypoxia: an alarm signal during intestinal inflammation. Nat Rev Gastroenterol Hepatol 2010;7:281-287.
21 Taylor CT, Colgan SP: Hypoxia and gastrointestinal disease. J Mol Med (Berl) 2007;85: 1295-1300.

22 Pollard TD, Borisy GG: Cellular motility driven by assembly and disassembly of actin filaments. Cell 2003;112:453-465.

23 Borregaard N, Herlin T: Energy metabolism of human neutrophils during phagocytosis. J Clin Invest 1982;70:550-557.

24 Gabig TG, Bearman SI, Babior BM: Effects of oxygen tension and $\mathrm{pH}$ on the respiratory burst of human neutrophils. Blood 1979;53: 1133-1139.

25 Semenza GL: Life with oxygen. Science 2007; 318:62-64.

26 Kaelin WG Jr, Ratcliffe PJ: Oxygen sensing by metazoans: the central role of the HIF hydroxylase pathway. Mol Cell 2008;30:393-402.

27 Fandrey J, Gorr TA, Gassmann M: Regulating cellular oxygen sensing by hydroxylation. Cardiovasc Res 2006;71:642-651.

28 Greer SN, Metcalf JL, Wang Y, Ohh M: The updated biology of hypoxia-inducible factor. EMBO J 2012;31:2448-2460.

29 Bosco MC, Puppo M, Blengio F, Fraone T, Cappello P, Giovarelli M, et al: Monocytes and dendritic cells in a hypoxic environment: spotlights on chemotaxis and migration. Immunobiology 2008;213:733-749.

30 Brahimi-Horn MC, Chiche J, Pouysségur J: Hypoxia signalling controls metabolic demand. Curr Opin Cell Biol 2007;19:223-229.

31 Franke K, Gassmann M, Wielockx B: Erythrocytosis: the HIF pathway in control. Blood 2013;122:1122-1128.

32 Mastrogiannaki M, Matak P, Keith B, Simon MC, Vaulont S, Peyssonnaux C: HIF-2alpha, but not HIF-1alpha, promotes iron absorption in mice. J Clin Invest 2009;119:1159-1166.

33 Nizet V, Johnson RS: Interdependence of hypoxic and innate immune responses. Nat Rev Immunol 2009;9:609-617.

34 Pialoux V, Mounier R, Brown AD, Steinback CD, Rawling JM, Poulin MJ: Relationship between oxidative stress and HIF-1 alpha mRNA during sustained hypoxia in humans. Free Radic Biol Med 2009;46:321-326.

35 Semenza GL: Oxygen sensing, homeostasis, and disease. N Engl J Med 2011;365:537-547.

36 Wang Q, Liu C, Zhu F, Liu F, Zhang P, Guo C, et al: Reoxygenation of hypoxia-differentiated dentritic cells induces Th1 and Th17 cell differentiation. Mol Immunol 2010;47:922-931. 
37 Vavricka SR, Rogler G: New insights into the pathogenesis of Crohn's disease: are they relevant for therapeutic options? Swiss Med Wkly 2009;139:527-534.

38 Vavricka SR, Rogler G: Recent advances in the etiology and treatment of Crohn's disease. Minerva Gastroenterol Dietol 2010;56:203211.

39 Rogler G, Vavricka S: Exposome in IBD: recent insights in environmental factors that influence the onset and course of IBD. Inflamm Bowel Dis 2015;21:400-408.

40 Chen LW, Egan L, Li ZW, Greten FR, Kagnoff MF, Karin M: The two faces of IKK and NFkappaB inhibition: prevention of systemic inflammation but increased local injury following intestinal ischemia-reperfusion. Nat Med 2003;9:575-581.
41 Kles KA, Tappenden KA: Hypoxia differentially regulates nutrient transport in rat jejunum regardless of luminal nutrient present. Am J Physiol Gastrointest Liver Physiol 2002; 283:G1336-G1342.

42 Kles KA, Wallig MA, Tappenden KA: Luminal nutrients exacerbate intestinal hypoxia in the hypoperfused jejunum. JPEN J Parenter Enteral Nutr 2001;25:246-253.

43 Carra GE, Ibáñez JE, Saraví FD: Electrogenic transport, oxygen consumption, and sensitivity to acute hypoxia of human colonic epithelium. Int J Colorectal Dis 2011;26:1205-1210.

44 Eltzschig HK, Carmeliet P: Hypoxia and inflammation. N Engl J Med 2011;364:656-665.
45 Vavricka SR, Rogler G, Maetzler S, Misselwitz B, Safroneeva E, Frei P, et al: High altitude journeys and flights are associated with an increased risk of flares in inflammatory bowel disease patients. J Crohns Colitis 2014;8:191199.

46 Code of Federal Regulations: Title 14: Part 25.841. Washington Government Printing Office, 1986.

47 Cottrell JJ: Altitude exposures during aircraft flight. Flying higher. Chest 1988;93:81-84.

48 British Thoracic Society Standards of Care Committee: Managing passengers with respiratory disease planning air travel: British thoracic society recommendations. Thorax 2002 ; 57:289-304.

49 Tzani P, Pisi G, Aiello M, Olivieri D, Chetta A: Flying with respiratory disease. Respiration 2010;80:161-170. 